\title{
Cations But Not Anions Regulate the Responsiveness of Kainate Receptors
}

\author{
David M. MacLean, Adrian Y. C. Wong, Anne-Marie Fay, and Derek Bowie \\ Department of Pharmacology and Therapeutics, McGill University, Montréal, Québec, Canada H3G OB1
}

\begin{abstract}
Kainate-selective ionotropic glutamate receptors are unique among ligand-gated ion channels in their obligate requirement of external anions and cations for activation. Although it is established that the degree of kainate receptor (KAR) activation is shaped by the chemical nature of the agonist molecule, the possible complementary role of external ions has yet to be examined. Here we show that external cations but not anions regulate the responsiveness to a range of full and partial agonists acting on rat GluK2 receptors. This observation is unexpected as previous work has assumed anions and cations affect KARs in an identical manner through functionally coupled binding sites. However, our data demonstrate that anion- and cation-binding pockets behave discretely. We suggest cations uniquely regulate a pregating or flipping step that impacts the closed-cleft stability of the agonist-binding domain (ABD). This model departs from a previous proposal that KAR agonist efficacy is governed by the degree of closure elicited in the ABD by ligand binding. Our findings are, however, in line with recent studies on Cys-loop ligand-gated ion channels suggesting that the "flipping" mechanism has been conserved by structurally diverse ligand-gated ion channel families as a common means of regulating neurotransmitter behavior.
\end{abstract}

\section{Introduction}

The concept that the chemical nature of a neurotransmitter is a key determinant of efficacy has long been supported by extensive structure-activity studies of many ligand-gated ion channels. At AMPA- and kainate (KA)-type ionotropic glutamate receptors (iGluRs), initial studies proposed that agonist efficacy resides in the conformations adopted by the agonist-binding domain $(\mathrm{ABD})$ with full agonists more effective at promoting domain closure than partial agonists (Armstrong and Gouaux, 2000; Jin et al., 2003; Mayer, 2005). However, this long-held view has been challenged by more recent work on AMPA receptors (AMPARs) (Zhang et al., 2006, 2008) and kainate receptors (KARs) (Fay et al., 2009; Frydenvang et al., 2009) that has failed to report a clear relationship between agonist efficacy and domain closure. From their work on AMPARs, Zhang et al. $(2006,2008)$ have proposed that agonist efficacy is determined by the stability of the closedcleft conformation of the ABD. Whether this mechanism also accounts for agonist behavior at KARs has yet to be established. An additional complication is that KARs exhibit an absolute requirement for external anions and cations as well as the neurotransmitter L-glutamate (L-Glu) for activation (Wong et al., 2006). Consequently, it is possible that the degree of KAR activa-

\footnotetext{
Received Aug. 17, 2010; revised Nov. 30, 2010; accepted Dec. 6, 2010.

This work was supported by an operating grant from the Canadian Institutes for Health Research (CIHR). D.M.M. was supported by a Best \& Banting CIHR doctoral fellowship, and D.B. is the recipient of the Canada Research Chair award in Receptor Pharmacology. We are grateful to Drs. Greg Miller and Phillip Biggin for providing a thoughtfu critique of this manuscript and to Julien Ollivier for providing us with Facile version 0.25 and previous versions. We give special thanks to Mark Aurousseau for discussions on the structural basis of cation modulation.

Correspondence should be addressed to Dr. Derek Bowie, Department of Pharmacology and Therapeutics, McGill University, 3655 Promenade Sir William 0sler, Montreal, Québec, Canada H3G 0B1. E-mail: derek.bowie@mcgill.ca. DOI:10.1523/JNEUROSCI.4314-10.2011

Copyright $\odot 2011$ the authors $\quad 0270-6474 / 11 / 312136-09 \$ 15.00 / 0$
}

tion is shaped not only by the agonist molecule but also by external ions.

To examine this, we have performed experiments to test whether KAR agonist efficacy is affected by the composition of the external anion and/or cation environment. Unexpectedly, we find that cations, but not anions, regulate the responsiveness of GluK2 (formerly GluR6) KARs to a wide range of full and partial agonists. Simulations with KAR gating models suggest that cations achieve this by influencing the closed-cleft stability of the $\mathrm{ABD}$ as described for agonist behavior at AMPA-type iGluRs (Zhang et al., 2006, 2008). Together with anion and cation effects on dimer stability (Chaudhry et al., 2009), this mechanism explains how the behavior of KARs is shaped by both the chemical nature of the agonist molecule and the ions present in the external milieu.

\section{Materials and Methods}

Cell culture and transfection. HEK tsA201 cells were transiently cotransfected with cDNA encoding wild-type or mutant GluK2(Q) KAR subunits and enhanced green fluorescent protein $\left(\mathrm{eGFP}_{\mathrm{S65T}}\right)$ as described previously (Bowie, 2002). After transfection for 6-10 h using the calcium phosphate precipitation method, cells were washed twice with divalentcontaining PBS and maintained in fresh medium (MEM containing 10\% FBS, Glutamax, penicillin, and streptomycin). Electrophysiological recordings were performed 24-48 h later.

Electrophysiology. All experiments were performed on outside-out patches excised from transfected cells using thin-walled borosilicate glass pipettes (2-6 M $\Omega$ ) coated with dental wax. Agonist solutions were rapidly applied for $250 \mathrm{~ms}$ using a piezo-stack-driven perfusion system. Sufficient time between applications was allowed for complete recovery from macroscopic desensitization [ $15 \mathrm{~s}$ for Glu, L-cysteic acid (Cys), and L-aminoadipate (AA); $60 \mathrm{~s}$ for KA; $180 \mathrm{~s}$ for Domoate (Dom)]. The solution exchange time was determined routinely at the end of each experiment by measuring the liquid junction current (10-90\% rise time, 50-200 $\mu \mathrm{s}$ ). All recordings were performed at room temperature using an Axopatch 200B amplifier (Molecular Devices) with current records filtered 


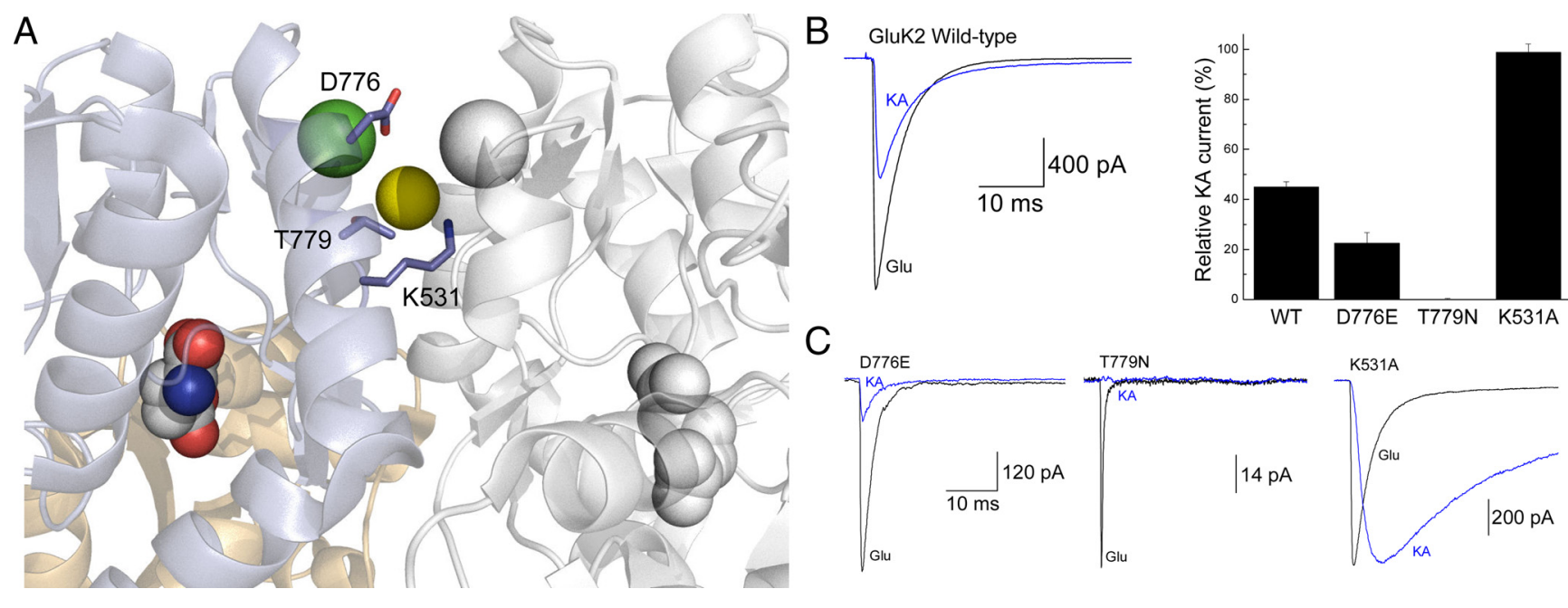

Figure 1. Mutation of GluK2 dimer interface KA efficacy. $A$, Crystal structure of the dimeric GluK2 ligand-binding domain in complex with Glu and two cations (one green and one white sphere) and one anion (yellow sphere) (Protein Data Bank number 3G3F). B, Left, Representative response of wild-type (WT) GluK2 receptors to Glu (10 mm; black trace) and KA (1 mm; blue trace; patch number 030724p2). Right, Plot summarizing the effect of point mutations on the relative responsiveness of GluK2 receptors to KA. C, Membrane currents elicited by Glu ( $10 \mathrm{~mm}$; black traces) and KA (1 mм; blue traces) for GluK2 mutants D776E (left; patch number 100121p6), T779N (middle; patch number 100128p2), and K531A (right; patch number 070215p2).

at $10 \mathrm{kHz}$ and digitized at $50-100 \mathrm{kHz}$. The reference electrode was connected to the bath via an agar bridge of $3 \mathrm{~m} \mathrm{KCl}$. Series resistances (3-15 M $\Omega$ ) were routinely compensated by $95 \%$. Data were acquired using pClamp9 software (Molecular Devices) and illustrated using Origin 7 (OriginLab).

Solutions. External solutions contained $150 \mathrm{~mm} X Y, 5$ mм HEPES, 0.1 $\mathrm{mm} \mathrm{CaCl}, 0.1 \mathrm{~mm} \mathrm{MgCl}_{2}$, and $2 \%$ phenol red, where $X$ is $\mathrm{Na}^{+}, \mathrm{K}^{+}$, or $\mathrm{Rb}+$ and $Y$ is $\mathrm{Cl}^{-}, \mathrm{I}^{-}$, or $\mathrm{NO}_{3}^{-}$. The osmotic pressure was adjusted to 290-295 mOsm using sucrose, and the $\mathrm{pH}$ was adjusted to 7.4 with the appropriate hydroxide (e.g., $\mathrm{KOH}$ for $\mathrm{KCl}$ ). The internal solution contained (in mM) $115 \mathrm{NaCl}, 10 \mathrm{NaF}, 5$ HEPES, $5 \mathrm{Na}_{4}$ BAPTA, $0.5 \mathrm{CaCl}_{2}, 1$ $\mathrm{MgCl}_{2}$, and $10 \mathrm{Na}_{2} \mathrm{ATP}$ to chelate endogenous polyamines; $\mathrm{pH}$ was adjusted to 7.3 with $5 \mathrm{~N} \mathrm{NaOH}$, and the osmotic pressure was adjusted to correspond with external solutions. Agonist solutions were prepared by dissolving the agonist in the appropriate external solution and adjusting the $\mathrm{pH}$ with the corresponding hydroxide solution. Agonist concentrations were selected as determined by Fay et al. (2009), made from concentrated $10 \times$ stock solutions, stored at $-20^{\circ}$, and thawed before use.

Data analysis. Data were analyzed using Clampfit 9.0 and tabulated using Excel (Microsoft Corp). To compare the effect of external anions or cations on responses elicited by a range of different GluK2 receptor agonists, peak responses were expressed relative to the full agonist, L-Glu (e.g., $\mathrm{KA}$ in $\mathrm{KCl}$ vs $\mathrm{L}-\mathrm{Glu}$ in $\mathrm{KCl}$ in the same recording). To denote this, we have used the terms "relative efficacy" or "responsiveness" in the text.

Dose-response data were normalized, pooled across patches, and fit with the logistic equation of the following form:

$$
I_{(x)}=I_{\max } /\left(1+\left(\mathrm{EC}_{50} / x\right)^{n}\right),
$$

where $I_{(x)}$ is the normalized peak current at agonist concentration $x, I_{\max }$ is the interpolated maximal response, and $n$ is the slope.

The peak and equilibrium response relationship in Figure 7 was fit with the following equation:

$$
S S=k /\left(\operatorname{Peak}^{n}\right)+C,
$$

where $S S$ is the amplitude of the equilibrium response divided by the peak response, Peak is the peak response of an agonist as a percentage of the Glu peak response, and $C$ is the minimal predicted equilibrium response. Initial fits with a simple inverse relationship (i.e., $S S=1 /$ Peak) were never able to satisfactorily match the data, necessitating factors $k$ and $n$ to allow the fit to better match the data. The fit values are not intended to reveal any mechanistic insight (other than the inverse relationship between efficacy and equilibrium activation) but to highlight the trend that the data follow an approximate inverse relationship. When required, data were tested for statistical significance using a two-tailed, paired Student's $t$ test. A $p$ value of $\leq 0.05$ was considered statistically significant.

Kinetic simulations. Simulations of peak and equilibrium responses for the three kinetic models tested (see Fig. 8) were performed using rate constants published previously (Bowie et al., 1998; Zhang et al., 2008; Perrais et al., 2009). The software tool FACILE version 0.25 (courtesy of J. Ollivier, McGill University, Montreal, Canada) (Siso-Nadal et al., 2007) was used to convert the differential equations for each kinetic scheme into Matlab (version 7.8; MathWorks) format. A purpose-written Matlab code was then used to run the simulations and plot the results. For each simulation, the specified rate constant was altered in half log-unit steps (i.e., $1 \mathrm{x}, 3 \mathrm{x}, 10 \mathrm{x}, 30 \mathrm{x}$, etc.) across 5 orders of magnitude.

\section{Results}

\section{Disruption to the dimer interface affects} agonist responsiveness

The structural basis of agonist efficacy at KA-type iGluRs is still unknown. Until recently, it had been assumed that KARs behave much like AMPARs where agonist efficacy is thought to be determined by the extent of closure elicited by ligand binding to the ABD (Armstrong and Gouaux, 2000; Jin et al., 2003). In this model, full agonists are more effective activators than partial agonists because they promote greater domain closure. However, more recent work on both AMPARs and KARs has questioned the validity of this idea since a clear relationship between agonist efficacy and the extent of domain closure was not able to be established (Zhang et al., 2008; Fay et al., 2009; Frydenvang et al., 2009; Birdsey-Benson et al., 2010). Given this, we reasoned that regions outside the agonistbinding pocket, such as the dimer interface, may have a more prominent role in fine tuning agonist efficacy.

To examine this, we compared the effect of disrupting the dimer interface of GluK2 receptors on the responses to the full and partial agonists, L-Glu and KA (Fig. 1). We purposely mutated amino acid residues within the vicinity of the anion- and cation-binding pockets (Fig. $1 A$ ) to test a suggestion proposed in a previous study from our laboratory that external ions may regulate KAR agonist efficacy (Fay et al., 2009). Consistent with this hypothesis, all three mutants, D776E, T779N, and K531A, significantly affected the agonist responsiveness of the GluK2 receptor (Fig. $1 B, C$ ). Compared with L-Glu, peak responses elicited by KA were either greatly diminished by the D776E mutant or com- 
pletely abolished with T779N (Fig. 1C, right and middle). In contrast, neutralization of the positively charged Lys residue at position 531 with an Ala dramatically increased the peak amplitude of KA-evoked currents to match that of L-Glu (Fig. 1C, right). Together, these data agree with a previous study (Fleck et al., 2003) that modifications to the dimer interface can significantly impact the strength of KAR agonists. Given the close proximity of each mutation to the anion- and cation-binding sites, we planned additional experiments to examine the possible role of external ions in regulating KAR agonist efficacy.

\section{External anions do not affect the responsiveness to kainate receptor agonists}

Previous work from our laboratory and others has shown that external anions and cations exert an apparently concomitant effect on both the peak amplitude and decay kinetics of the response elicited by the neurotransmitter L-Glu (Bowie, 2010). For example, compared with other alkali metal ions, L-Glu responses in external $\mathrm{Na}^{+}$are larger in amplitude and deactivate and desensitize more slowly (Bowie, 2002). Similar findings were reported when external $\mathrm{Cl}^{-}$is replaced by other halide ions (Bowie, 2002). Together with the fact that both anions and cations similarly regulate the stability of the interface between KAR subunits (Chaudhry et al., 2009), it has been assumed that both ion species regulate KARs through a common mechanism. An important caveat, however, is that the effect of external ions on GluK2 receptor responses has not been extended to other agonists. Given this, we examined the responsiveness of a series of L-Glu analogs (Fay et al., 2009) in solutions with different external anions or cations.

Figure 2 shows typical GluK2 agonist responses observed in the presence of equimolar sodium salt (i.e., $150 \mathrm{~mm}$ ) solutions of chloride $\left(\mathrm{Cl}^{-}\right)$, nitrate $\left(\mathrm{NO}_{3}^{-}\right)$, and iodide $\left(\mathrm{I}^{-}\right)$. In all cases, rapid application of saturating concentrations of either L-Glu (10 mM) or KA ( $1 \mathrm{~mm})$ resulted in fast-activating responses that desensitized almost completely (Fig. 2A). As expected from previous work, complete replacement of external $\mathrm{Cl}^{-}$with either $\mathrm{NO}_{3}^{-}$or $\mathrm{I}^{-}$accelerated the onset of L-Glu macroscopic desensitization by about twofold to threefold (Table 1) with a concomitant reduction in peak response amplitude (Bowie, 2002). In $150 \mathrm{mM} \mathrm{Cl}^{-}$, peak KA responses were slightly less than half $(44.9 \pm 2.2 \%)$ (Table 1) of that elicited by L-Glu (Fig. $2 \mathrm{~A}$, left), consistent with its reported role as a partial agonist (Fay and Bowie, 2006; Fay et al., 2009). Substituting $\mathrm{Cl}^{-}$with either $\mathrm{NO}_{3}^{-}$or $\mathrm{l}^{-}$did not alter the responsiveness to KA since peak responses were $43.5 \pm 2.2 \%$ $(n=8)$ and $39.9 \pm 2.5 \%(n=4)$, respectively, of the L-Glu response (Fig. $2 A$, middle and right; Table 1 ). Similar findings were observed when we extended this observation to the full agonist, Cys (40 mM), and partial agonists, AA (40 mM) and Dom $(50 \mu \mathrm{M})$, demonstrating that the external anion composition does not affect agonist responsiveness (Fig. 2 B, Table 1). In support of this, the scatter plot of the relationship between peak agonist responses observed in external $\mathrm{Cl}^{-}$versus responses observed in either $\mathrm{NO}_{3}^{-}$or $\mathrm{l}^{-}$were well fit by a linear regression function with a slope close to unity (Fig. $2 \mathrm{~B}$, dotted lines) $\left(\mathrm{NO}_{3}^{-}\right.$: $\left.m=0.96 \pm 0.01, r^{2}=0.99 ; \mathrm{I}^{-}: m=0.89 \pm 0.01, r^{2}=0.99\right)$.

\section{Cations regulate the responsiveness to kainate receptor agonists}

Contrary to our observation with anions, external cations had a pronounced effect on the responsiveness of membrane currents elicited by KA relative to L-Glu (Fig. 3). In external $150 \mathrm{mM} \mathrm{Na}^{+}$, peak KA responses were $44.9 \pm 2.2 \%(n=13)$ (Table 1$)$ relative

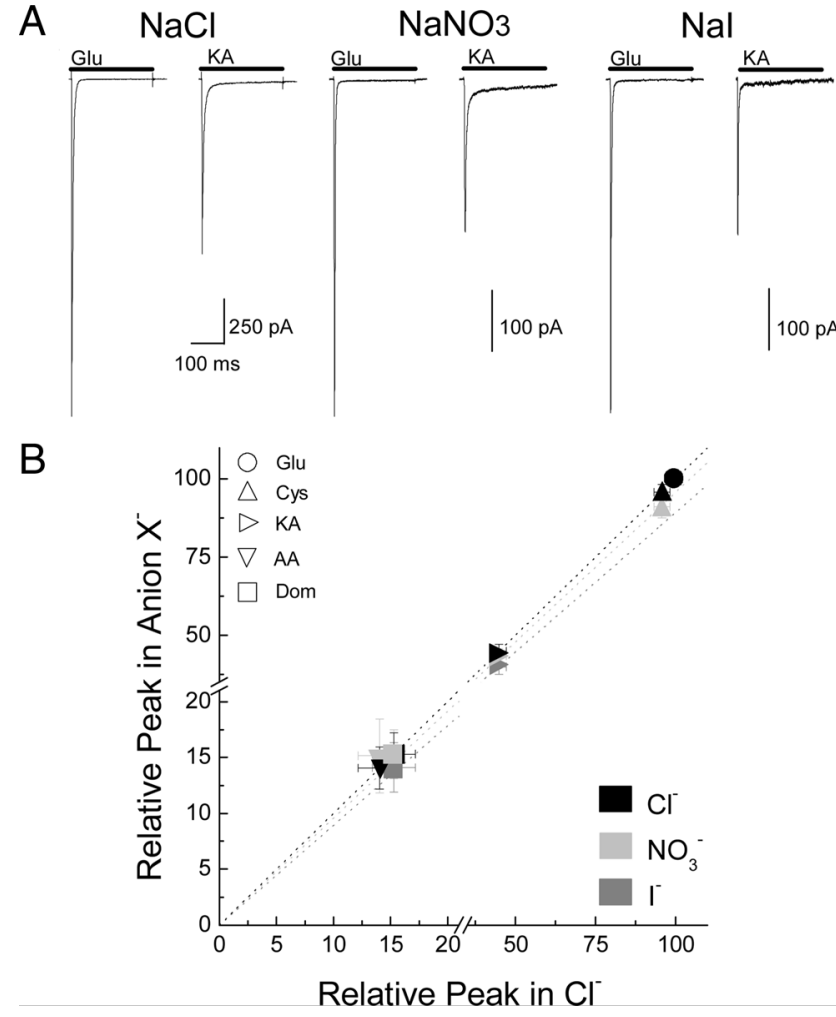

Figure 2. Anions do not affect responsiveness to GluK2 agonists. $A$, Typical membrane currents evoked by GluK2 receptors in response to Glu (10 mM) or KA (1 mM) in $150 \mathrm{~mm} \mathrm{NaCl}$ (left; patch number 030724p2), $\mathrm{NaNO}_{3}$ (middle; patch number 051215p3), or Nal (right; patch number 060210p3). B, Summary plot comparing the relative peak response produced by each agonist in different external anion solutions [ $\mathrm{NaCl}$ (black), $\mathrm{NaNO}_{3}$ (light gray), or Nal (dark gray)]. Each dotted line corresponds to a linear regression fit of the data obtained in different external anion solutions.

to L-Glu. However, replacement of $\mathrm{Na}^{+}$with equimolar concentrations of either $\mathrm{K}^{+}$or $\mathrm{Rb}^{+}$caused a twofold reduction in the responsiveness to KA to $19.8 \pm 2.6 \%(n=6)$ or $16.6 \pm 2.0 \%(n=$ 3 ) of the peak L-Glu response, respectively (Fig. $3 A$, Table 1). Similarly, in $150 \mathrm{~mm}$ external $\mathrm{Na}^{+}$, peak AA responses were $14.0 \pm 1.9 \%(n=13)$ of the L-Glu response. However, the responsiveness to AA declined again about twofold to $6.7 \pm 1.4 \%$ $(n=4)$ or $9.4 \pm 1.8 \%(n=3)$ of the peak L-Glu response in $\mathrm{K}^{+}$ or $\mathrm{Rb}+$, respectively (Fig. $3 B$ ). As expected, the relationship between peak agonist responses observed in external $\mathrm{Na}^{+}$versus responses observed in either $\mathrm{K}^{+}$or $\mathrm{Rb}+$ were well fit by a linear regression function with slopes substantially lower than unity (Fig. 3B, dotted lines) $\left(\mathrm{K}^{+}: m=0.44 \pm 0.01, r^{2}=0.99\right.$; $\mathrm{Rb}+$ : $\left.m=0.35 \pm 0.03, r^{2}=0.93\right)$.

Together, these findings show for the first time that anions and cations have distinct and separable effects on KARs. We were, however, concerned that cation- and/or anion-binding sites may not be saturated at the ion concentrations used in our experiments (Bowie, 2002; Plested and Mayer, 2007; Plested et al., 2008). A specific concern was that incomplete occupancy was more relevant to cations since the anion site has been proposed to be closer to saturation at physiological (i.e., $150 \mathrm{~mm} \mathrm{NaCl}$ ) salt levels (Plested et al., 2008). Given this, it was possible that the different effects of cations on agonist responsiveness may simply reflect a lower occupancy of their binding site. To test this, we repeated our experiments with L-Glu, KA, and AA in $600 \mathrm{~mm}$ $\mathrm{NaCl}$ and $600 \mathrm{~mm} \mathrm{KCl}$ (Fig. 4). We reasoned that using solutions 
Table 1. Summary of GluK2 responses to various agonists in differing ionic conditions

\begin{tabular}{|c|c|c|c|c|c|}
\hline Agonist & Ion & Number & $\begin{array}{l}\text { Relative } \\
\text { peak }\end{array}$ & $\tau_{\text {fast }}$ & $\%$ Equilibrium \\
\hline \multirow[t]{5}{*}{ Glu (10 mм) } & $\mathrm{NaCl}$ & 13 & 100 & $4.9 \pm 0.2$ & $0.31 \pm 0.04$ \\
\hline & $-\mathrm{NO}_{3}$ & 10 & 100 & $2.2 \pm 0.1$ & $1.1 \pm 0.2$ \\
\hline & -1 & 4 & 100 & $2.2 \pm 0.04$ & $0.2 \pm 0.002$ \\
\hline & K- & 6 & 100 & $1.2 \pm 0.1$ & $0.3 \pm 0.1$ \\
\hline & $\mathrm{Rb}-$ & 5 & 100 & $0.6 \pm 0.1$ & $0.6 \pm 0.3$ \\
\hline \multirow[t]{4}{*}{ Cys (40 mm) } & $\mathrm{NaCl}$ & 13 & $95.8 \pm 2.5$ & $3.0 \pm 0.2$ & $0.6 \pm 0.1$ \\
\hline & $\begin{array}{l}-\mathrm{NO}_{3} \\
-\mathrm{I}\end{array}$ & 8 & $91.1 \pm 3.4$ & $1.4 \pm 0.1$ & $4.9 \pm 0.6$ \\
\hline & K- & 5 & $64.4 \pm 3.8$ & $0.8 \pm 0.05$ & $1.0 \pm 0.3$ \\
\hline & $\mathrm{Rb}-$ & 3 & $32.3 \pm 1.6$ & $0.5 \pm 0.1$ & $1.1 \pm 0.3$ \\
\hline \multirow[t]{5}{*}{ KA (1 mM) } & $\mathrm{NaCl}$ & 13 & $44.9 \pm 2.2$ & $4.8 \pm 0.2$ & $1.8 \pm 0.4$ \\
\hline & $-\mathrm{NO}_{3}$ & 8 & $43.5 \pm 2.2$ & $3.5 \pm 0.2$ & $3.2 \pm 1.3$ \\
\hline & -1 & 4 & $39.9 \pm 2.5$ & $4.5 \pm 0.4$ & $2.4 \pm 0.4$ \\
\hline & K- & 6 & $19.8 \pm 2.6$ & $1.7 \pm 0.3$ & $3.1 \pm 0.6$ \\
\hline & $\mathrm{Rb}-$ & 3 & $16.6 \pm 2.0$ & $3.2 \pm 0.2$ & $3.4 \pm 1.6$ \\
\hline \multirow{4}{*}{$\mathrm{AA}(40 \mathrm{~mm})$} & $\mathrm{NaCl}$ & 13 & $14.0 \pm 1.9$ & $13.2 \pm 0.7$ & $11.1 \pm 1.4$ \\
\hline & $\begin{array}{l}-\mathrm{NO}_{3} \\
-\mathrm{I}\end{array}$ & 5 & $14.1 \pm 3.3$ & $4.7 \pm 0.7$ & $14.1 \pm 3.1$ \\
\hline & K- & 4 & $6.7 \pm 1.4$ & $3.8 \pm 0.2$ & $11.4 \pm 4.1$ \\
\hline & $\mathrm{Rb}-$ & 3 & $9.4 \pm 1.8$ & $1.0 \pm 0.3$ & $3.3 \pm 1.4$ \\
\hline \multirow[t]{5}{*}{$\operatorname{Dom}(50 \mu \mathrm{M})$} & $\mathrm{NaCl}$ & 8 & $15.3 \pm 1.9$ & $16.9 \pm 4.5$ & $34.8 \pm 5.4$ \\
\hline & $-\mathrm{NO}_{3}$ & 4 & $15.3 \pm 2.2$ & $48.0 \pm 4.4$ & $72.2 \pm 5.7$ \\
\hline & -1 & 4 & $14.1 \pm 2.2$ & & $80.3 \pm 9.4$ \\
\hline & K- & 3 & $4.2 \pm 0.6$ & & $53.8 \pm 8.3$ \\
\hline & $\mathrm{Rb}-$ & 3 & $7.6 \pm 2.6$ & & $38.2 \pm 3.1$ \\
\hline
\end{tabular}

of high ionic strength would ensure that complete occupancy of anion- and/or cation-binding sites was achieved.

Despite these concerns, the responsiveness to both KA and AA relative to L-Glu remained unchanged. Peak KA- and AA-evoked responses in $600 \mathrm{mM} \mathrm{Na}^{+}$were $42.0 \pm 3.0 \%(n=4)$ and $18.3 \pm$ $2.0 \%(n=6)$ relative to L-Glu responses (Fig. 4$)$, which was in good agreement with our findings in $150 \mathrm{~mm} \mathrm{NaCl}$ (Table 1). Likewise, increasing $\mathrm{KCl}$ from 150 to $600 \mathrm{~mm}$ had little effect on responses elicited by KA $(23.6 \pm 2.4 \% ; n=6)$ but did exacerbate the differences between the responsiveness to AA $(4.7 \pm 2.0 \%$; $n=6$ ) relative to L-Glu (Fig. 4). Given this, we conclude that the decrease in responsiveness to $\mathrm{KA}$ and $\mathrm{AA}$ is not caused by an incomplete occupancy of the cation-binding site.

\section{Cations do not substantially alter agonist potency}

An additional concern was that replacing $\mathrm{Na}^{+}$with another cation may reduce the potency of $10 \mathrm{~mm} \mathrm{L-Glu}$ or $1 \mathrm{~mm}$ KA to such an extent that neither agonist has a maximal effect. To determine whether this is case, activation curves for both L-Glu and KA were constructed in equimolar (i.e., $150 \mathrm{mM}$ ) solutions of $\mathrm{Na}^{+}$and $\mathrm{K}^{+}$ and compared by fitting with the logistic equation (see Materials and Methods) to provide estimates of the agonist potency (i.e., $\mathrm{EC}_{50}$ ) and slope of the relationship (i.e., $\mathrm{nH}$ ) (Fig. 5). As revealed from the activation curves for L-Glu (Fig. 5B) and KA (Fig. 5D), replacing external $\mathrm{Na}^{+}$with $\mathrm{K}^{+}$exerted a modest rightward shift in the dose-response relationship but did not substantially reduce peak responses elicited by $10 \mathrm{~mm} \mathrm{L-Glu}$ and $1 \mathrm{~mm}$ KA to account for observations described in Figures 3 and 4. Membrane currents activated by L-Glu in external $\mathrm{Na}^{+}$had an $\mathrm{EC}_{50}$ of $450 \pm$ $40 \mu \mathrm{M}$ (Hill coefficient or $n_{\mathrm{H}}=1.1 \pm 0.1 ; n=4-5$ per data point $)$, which shifted to $870 \pm 110 \mu \mathrm{M}\left(n_{\mathrm{H}}=1.0 \pm 0.1 ; n=4-6\right.$ per data point) in external $\mathrm{K}^{+}$(Fig. $5 A, B$ ). Likewise, KA responses in external $\mathrm{Na}^{+}$exhibited an $\mathrm{EC}_{50}$ of $250 \pm 70 \mu \mathrm{M}$ $\left(n_{\mathrm{H}}=0.8 \pm 0.1 ; n=4-5\right.$ per data point $)$, which shifted to $480 \pm$
A

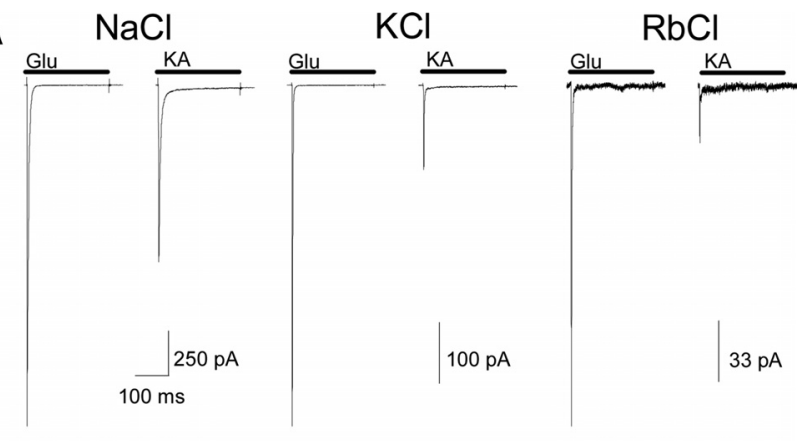

B

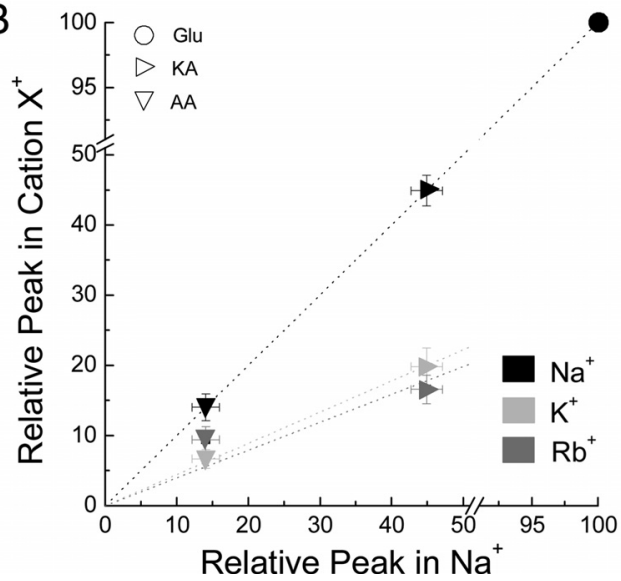

Figure 3. Cations profoundly affect responsiveness to GluK2 agonists. $\boldsymbol{A}$, Representative currents elicited by GluK2 receptors in response to Glu (10 mm) or KA (1 mm) in $150 \mathrm{~mm} \mathrm{NaCl}$ (left; patch number 030724p2), KCl (middle; patch number 051201p3), or RbCl (right; patch number 060502p1). B, Plot comparing the responsiveness to each agonist in different external cations relative to the response elicited by $10 \mathrm{~mm}$ Glu. Dotted lines show linear regression fits of the data in different external cations.

$90 \mu \mathrm{M}\left(n_{\mathrm{H}}=1.6 \pm 0.3 ; n=4-5\right.$ per data point $)$ in external $\mathrm{K}^{+}$ (Fig. 5C,D). Together, these data rule out the possibility of a substantial effect of the cation species on GluK2 agonist potency. Moreover, as described below, the entire effect of cations on GluK2 agonist responsiveness can be eliminated by disrupting cation binding.

\section{Cations regulate KAR agonist responses through the Met770 residue}

To determine whether this effect was mediated by the cationbinding site we and others have studied previously (Paternain et al., 2003; Wong et al., 2006, 2007; Plested et al., 2008), the agonist responsiveness of the GluK2 M770K receptor mutant was examined in different external cations (Fig. 6). Structural data have shown that this cation-binding site is composed of two backbone carbonyl groups (from Ile527 and Glu524) as well as two carboxyl side chains (from Glu524 and Asp528) (Plested et al., 2008; Chaudhry et al., 2009) (Fig. 6A, left). The "lid" of the cationbinding site is formed by Met770 (Fig. $6 \mathrm{~A}$ left). Importantly, replacement of the Met770 with a positively charged Lys (Fig. $6 A$, right), normally found in AMPARs, renders KARs insensitive to external cations through a mechanism involving both steric occlusion and electrostatic repulsion (Bowie, 2010).

As hypothesized, replacement of the Met770 residue with Lys completely removed the effect of external cations on GluK2 receptor agonist efficacy. The data summarizing this finding are shown in the scatter plots for GluK2 M770K (Fig. 6B, right) and the wild-type receptor (Fig. $6 B$, left). Note that we extended our 
observations to include two more ligands, the full agonist L-Cys and weak partial agonist Dom (Fig. $6 \mathrm{~B}$ ). Like KA and AA, the responsiveness to both agonists was significantly attenuated by replacing external $\mathrm{Na}^{+}$with $\mathrm{K}^{+}$or $\mathrm{Rb}+$ at wild-type receptors (Fig. 6B, left). Strikingly, L-Cys was converted from being a full agonist in 150 $\mathrm{mM} \mathrm{Na}^{+}(95.8 \pm 2.5 \%$ of L-Glu; $n=13)$ to a weak partial agonist in $150 \mathrm{mM} \mathrm{K}^{+}$ $(64.4 \pm 3.8 \%$ L-Glu; $n=5)$ and $\mathrm{Rb}+$ $(32.3 \pm 1.6 \%$ of L-Glu; $n=3)$ (Table 1$)$. In contrast, peak agonist responses elicited by GluK2 M770K receptors were entirely unaffected by the type of external cation present (Fig. 6B, right). For example, peak responses for $\mathrm{L}-\mathrm{Cys}$ in $\mathrm{Na}^{+}, \mathrm{K}^{+}$, or $\mathrm{Rb}+$ were $76.6 \pm 4.7 \%(n=6), 68.7 \pm$ $4.0 \%(n=4)$, or $76.0 \pm 2.0 \%(n=4)$, respectively (Fig. $6 B$, right). Likewise, the responsiveness of the weak partial agonist Dom was almost identical in $\mathrm{Na}^{+}(18.6 \pm$ $2.6 \%$ peak; $n=6), \mathrm{K}^{+}(26.9 \pm 5.0 \%$ peak; $n=4)$, and $\mathrm{Rb}+(22.4 \pm 6.0 \%$ peak; $n=$ 4) (Fig. $6 B$, right). Furthermore, the relationship between peak responses in external $\mathrm{Na}^{+}$versus either $\mathrm{K}^{+}$or $\mathrm{Rb}+$ for the M770K mutant were all well fit by linear regressions with slopes near unity (Fig. $6 \mathrm{~B}$, continuous lines) $\left(\mathrm{K}^{+}: m=0.97 \pm\right.$ $0.08, r^{2}=0.85 ; \mathrm{Rb}+: m=1.00 \pm 0.03$, $\left.r^{2}=0.98\right)$.

These data demonstrate that cations but not anions directly control the strength of KAR agonists. This effect is mediated through the same nonselective cationbinding site capped by the Met770 residue we and others have studied previously. Furthermore, this observation constitutes the first experimental dissociation of anion and cation effects on wild-type KARs and underscores the prominence of cations over anions in controlling gating behavior (Bowie, 2010).

\section{Cations regulate the equilibrium/peak response relationship}

How might cations regulate the responsiveness to KAR agonists? In the absence of a unified model of KAR gating, this issue cannot be resolved in a straightforward manner. Although kinetic models of KARs have been developed, in all cases, they are limited by the fact that they recapitulate only certain and not all aspects of KAR behavior (Bowie et al., 1998; Barberis et al., 2008). Consequently, speculation on how cations might work would be model dependent unless they were shown to affect a specific aspect of KAR behavior.

On this note, we have remarked previously (Fay and Bowie, 2006; Fay et al., 2009) of an unexpected relationship between the peak and equilibrium responses elicited by KAR agonists (Fig. 7). In descriptive terms, full agonists such as L-Glu evoke an equilibrium response that is small $(0.31 \pm 0.04 \%)$ (Table 1$)$ in amplitude relative to the peak response (Fig. $7 A$ ), whereas weak partial agonists, such as Dom, have a much larger equilibrium response

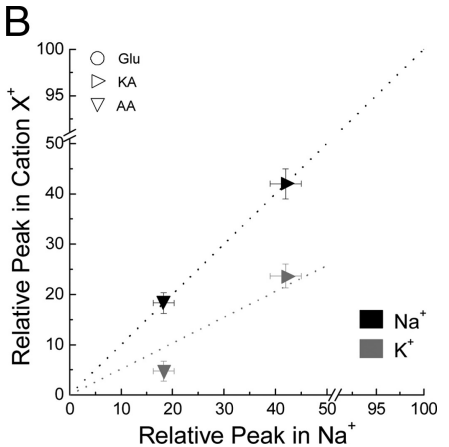

Figure 4. Cations regulate responsiveness to agonists even at saturating ionic conditions. $A$, Responses to Glu ( $10 \mathrm{mM}), \mathrm{KA}(1$ that in $\mathrm{KCl}$, the peak response to each partial agonist relative to $\mathrm{Glu}$ is reduced. $\boldsymbol{B}$, Summary plot showing averaged data of the same
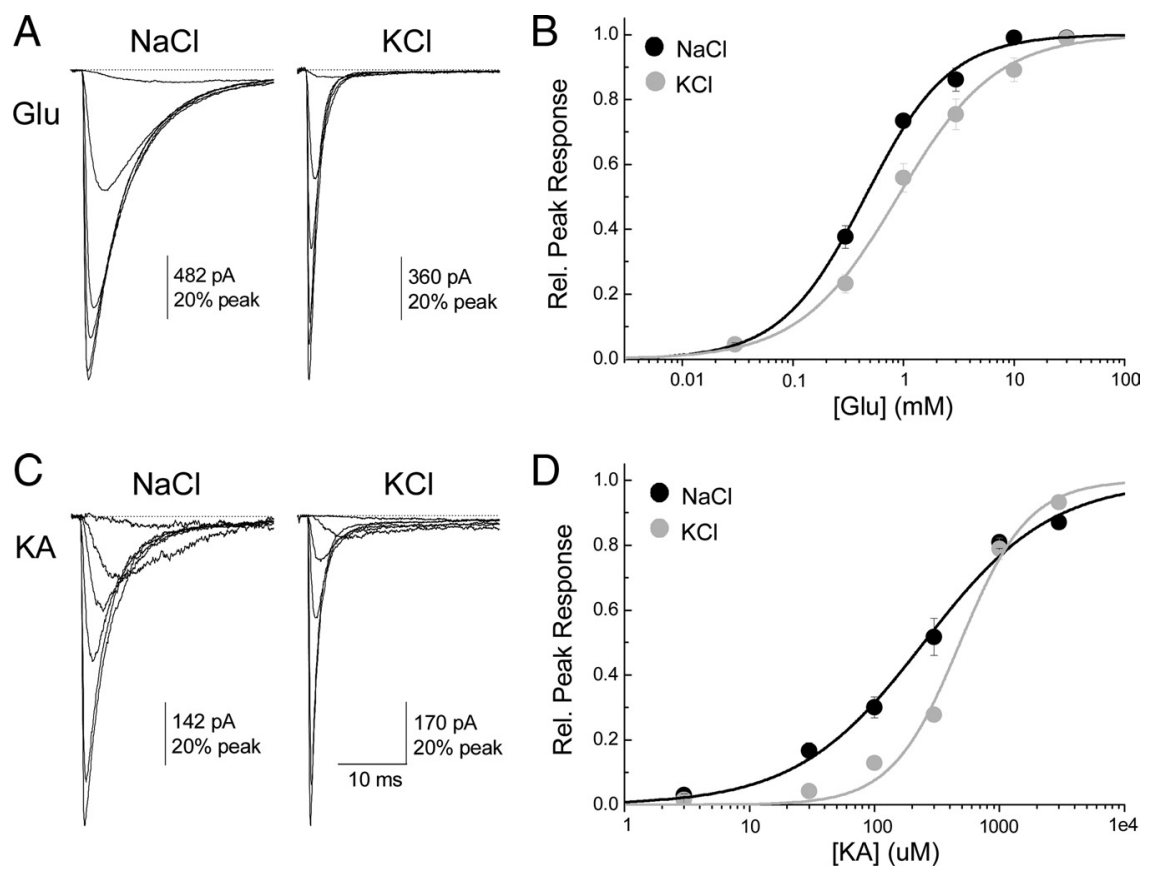

Figure 5. Cation substitution effects on Glu and KA dose-response curves. $A$, Membrane currents elicited by various concenresponse data and fits for $\mathrm{Glu}$ in $\mathrm{NaCl}$ (black circles and line) and $\mathrm{KCl}$ (gray circles and line). C, Membrane currents elicited by various concentrations of KA in $150 \mathrm{~mm} \mathrm{NaCl}$ (left; patch number 100927p5) or KCl (right; patch number 100928p5). D, Summary of dose-response data and fits for $\mathrm{KA}$ in $\mathrm{NaCl}$ (black circles and line) and $\mathrm{KCl}$ (gray circles and line).

$(34.8 \pm 5.4 \%)$ (Table 1$)$ relative to the peak (Fig. $7 A)$. Stated another way, full agonists elicit marked macroscopic desensitization (e.g., $>99 \%$ for $10 \mathrm{~mm}$ L-Glu in different cations) of GluK2 receptors, whereas partial agonists are much less effective in this regard (e.g., 30-65\% for $50 \mu \mathrm{m}$ Dom). Note that the term "macroscopic desensitization" is used here and throughout the text to denote only that responses decay in the agonist's presence. Consequently, the occurrence of macroscopic desensitization may be indicative of genuine microscopic desensitization but could also be explained by other mechanisms (see Discussion). Whatever the mechanism, this observation is unexpected in that there is no obvious reason, from our understanding of published gating models, to expect any correlation at all between the nature of the agonist (i.e., partial vs full) and the degree of macroscopic desensitization observed. Strikingly, this relationship extends across the entire agonist spectrum, from the five ligands used in 

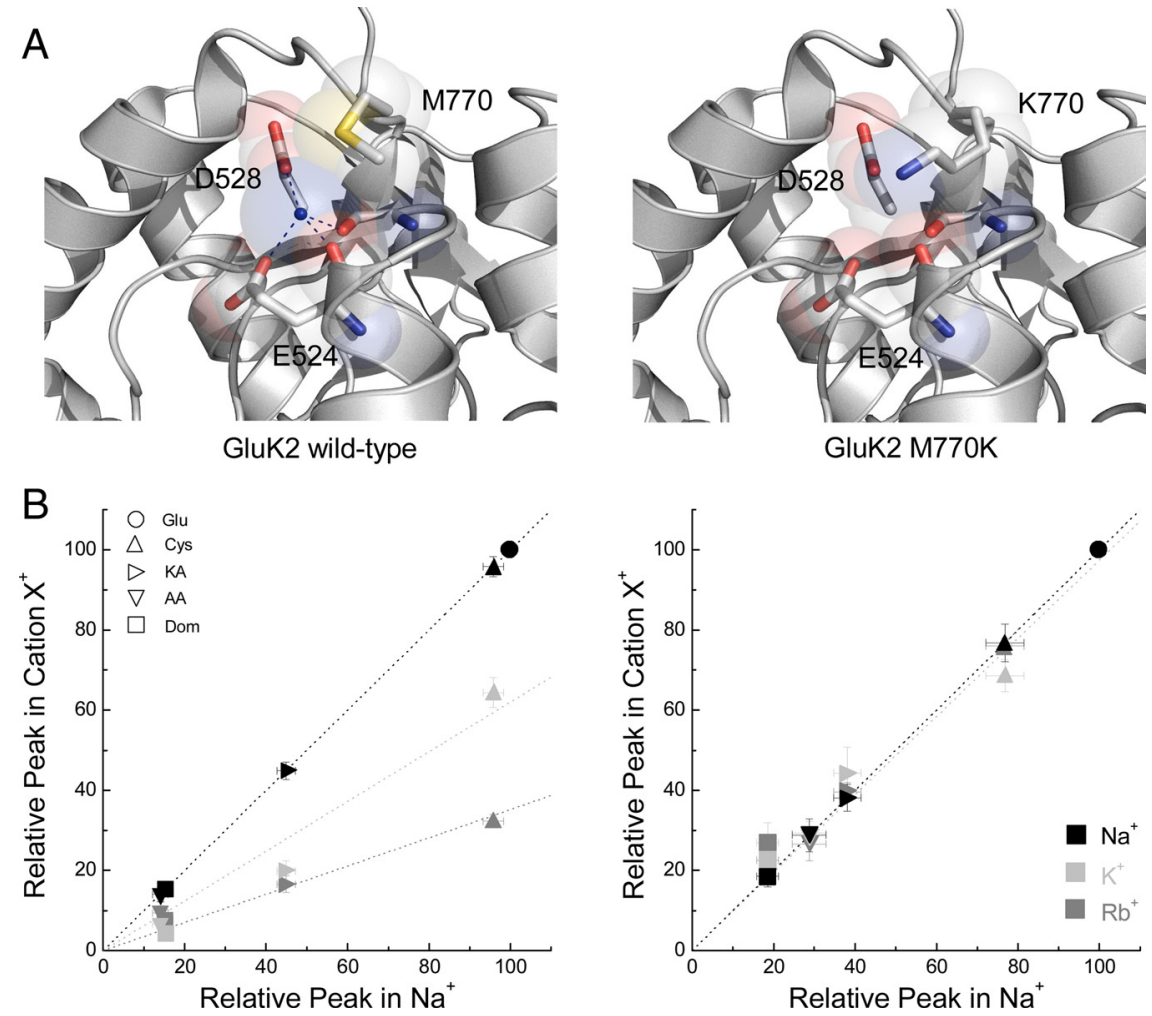

Figure 6. Tethering a positively charged Lys at the 770 residue eliminates cation regulation of GluK2. A, Left, Crystal structure of wild-type GluK2 cation-binding site showing residues that directly interact with the bound cation (blue sphere) (Protein Data Bank number 3G3F). Right, Identical structure as on the left, with the Met770 residue replaced by Lys. Note that the Lys side chain extends into the binding site preventing the docking of an external cation. $\boldsymbol{B}$, Left, Comparison of peak agonist responses (normalized to $\mathrm{Glu}$ in the indicated cation) in $\mathrm{NaCl}$ (black), $\mathrm{KCl}$ (light gray), and RbCl (dark gray) versus $\mathrm{NaCl}$ (abscissa) for all agonists tested on GluK2 wild-type receptors. Dotted lines show a linear regression fit of the data for each cation. Note that every agonist has a reduced peak response following cation substitution. Right, Similar comparison as shown on the left, but for GluK2 M770K receptors. Note in this case the effect of external cations on agonist peak responses is eliminated.

this study to all the other full and partial agonists we have described in previous work (Fig. 7B) (Fay et al., 2009). Moreover, it persists in different recording conditions including variations in the external cation and/or anion solutions (Fig. $7 B$ ), suggesting that this robust relationship is an intrinsic behavior of KARs.

Interestingly, when the predominant external cation, $\mathrm{Na}^{+}$, is exchanged for either $\mathrm{K}^{+}$or $\mathrm{Rb}+$, the equilibrium/peak response relationship becomes steeper (Fig. $7 B$, right, gray squares), an effect that is expected given our observations with external cations on peak agonist responses (Figs. 3-6). In contrast, the relationship is essentially unchanged with different anions (Fig. $7 B$, left, dark gray triangles), which again is entirely consistent with our data (Fig. 2). As discussed below, understanding this relationship may also provide an insight into how cations affect KARs. In broader terms, it also suggests a general mechanism for full and partial agonist behavior.

\section{Altering closed-cleft stability reproduces the equilibrium/ peak response relationship}

To understand the source of this inverse relationship and the effect of cations on it, we performed kinetic simulations with two commonly used gating schemes of KARs, namely the single-open state model (Bowie et al., 1998) and a more recent multi-open state model (Perrais et al., 2009) of gating. We also included a third model that corresponds to a new type of gating scheme, the pregating model, recently assigned to AMPARs (Zhang et al., 2008). Although this model is not intended to reproduce all fea- tures of KAR gating, it is nevertheless unique in that it includes a pregating step or flipping state (the transition from RA to $\mathrm{RA}^{\prime}$ ) (Fig. $8 \mathrm{~A}$ ) that explicitly models the dynamic equilibrium between the open and closed states of the ABD (Fig. $8 A$ ).

For each model, we began with published rate constants and, while keeping agonist affinity constant (i.e., not changing binding, $k$, or unbinding, $k_{-1}$, rates), altered gating reaction rates such as the rate of channel opening, $\beta$, or channel closure, $\alpha$, across 5 orders of magnitude to examine the entire range of agonist efficacy. We then examined how reducing efficacy using these parameters altered the equilibrium response. For every model, we found that changing gating parameters (i.e., reducing channel opening $\beta$, increasing channel closing $\alpha$, or entry into desensitization $\delta$ ) reduced the predicted peak response but did not reproduce the concomitant increase in the relative equilibrium response (Fig. $8 \mathrm{~B}$ ). However, in the case of the pregating model, increasing $\mathrm{ABD}$ cleft opening (CO; i.e., increasing rate constant) readily reduced efficacy in parallel with increasing the equilibrium response (Fig. $8 \mathrm{~B}$ ). This finding supports the idea that the stability of the closed cleft is an important element in partial agonism at non-NMDA receptors (NMDARs) (Robert et al., 2005; Zhang et al., 2006, 2008) as lowering closed-cleft stability (i.e., increasing $\mathrm{CO}$ ) mimics the inverse relationship we have observed across the agonist spectrum.

It is possible to approximate the equilibrium/peak response relationship by changing more than one parameter of the singleor multi-open state gating models. The simplest means of achieving this requires changing rates out of desensitization (i.e., $\gamma$ ) to regulate the equilibrium response while making concomitant changes in channel opening/closing rates (i.e., $\beta$ and $\alpha$ ) to obtain the desired peak response. This arrangement, however, implies a firm relationship between agonist efficacy and recovery from desensitization that is not supported by experimental work on KARs. For example, both AA and Dom are weak partial agonists producing $\sim 15 \%$ of the Glu peak in $\mathrm{NaCl}$. However, GluK2 channels activated by AA are fully recovered within $15 \mathrm{~s}$ (similar to Glu), whereas recovery from Dom requires $>120$ s (see Materials and Methods). Consequently, it is unlikely that the single- or multi-open state models can provide useful insight into our experimental findings. As a result, we conclude that alterations in the closed-cleft stability within the pregating model most aptly, and perhaps uniquely, account for the approximate inverse relationship between an agonist's peak and equilibrium response.

\section{Discussion}

This study reports two important findings that advance our understanding of KAR gating. First, we demonstrate an unexpected relationship between the nature of the agonist (i.e., partial vs full) and the degree of macroscopic desensitization. Importantly, a kinetic model that explicitly includes pregating steps reproduces 

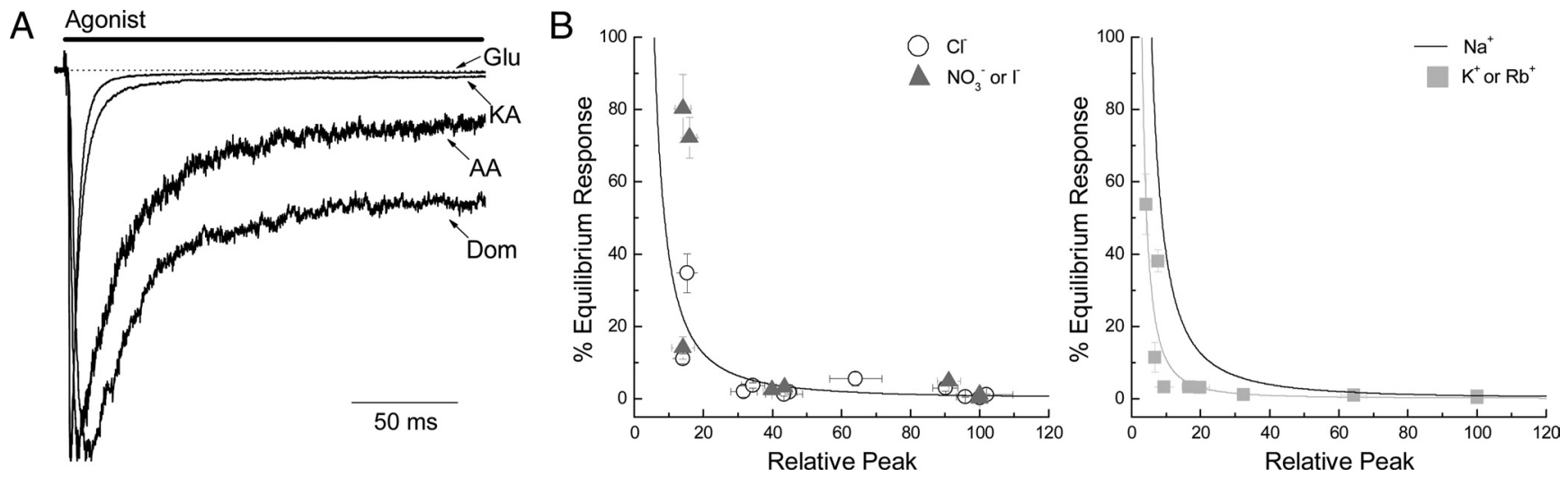

Figure 7. Agonist equilibrium/peak relationship. $A$, Membrane currents elicited by Glu ( $10 \mathrm{~mm}), \mathrm{KA}(1 \mathrm{~mm}), \mathrm{AA}(40 \mathrm{~mm})$, and Dom $(50 \mu \mathrm{m})$ in an outside-out patch expressing GluK2 receptors. Peak responses in each case were normalized to allow comparison (patch number 060207p2). $\boldsymbol{B}$, Left, Summary plot showing that there is an approximate inverse relationship between peak (abscissa) and equilibrium (ordinate axis) GluK2 agonist responses in $\mathrm{NaCl}$ (open circles) and in $\mathrm{Nal}_{\text {or }} \mathrm{NaNO}_{3}$ (gray triangles). Each point represents a specific agonist from this study (see Table 1) or from that by Fay et al. (2009). The solid line is a fit of the $\mathrm{NaCl}$ data using Eq. $2(n=1.67, \mathrm{k}=1866, \mathrm{C}=0.01)$. Right, Summary plot showing the inverse relationship in $\mathrm{NaCl}$ (black line from $\mathrm{NaCl}$ fit on the left) and in $\mathrm{KCl}$ or RbCl (light gray squares). The light gray line is a fit of the $\mathrm{K}^{+}$and $\mathrm{Rb}+$ data using $\mathrm{Eq} .2(n=1.8, \mathrm{k}=760, \mathrm{C}=0.1)$.

A Single-open state model

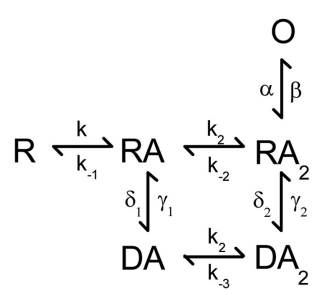

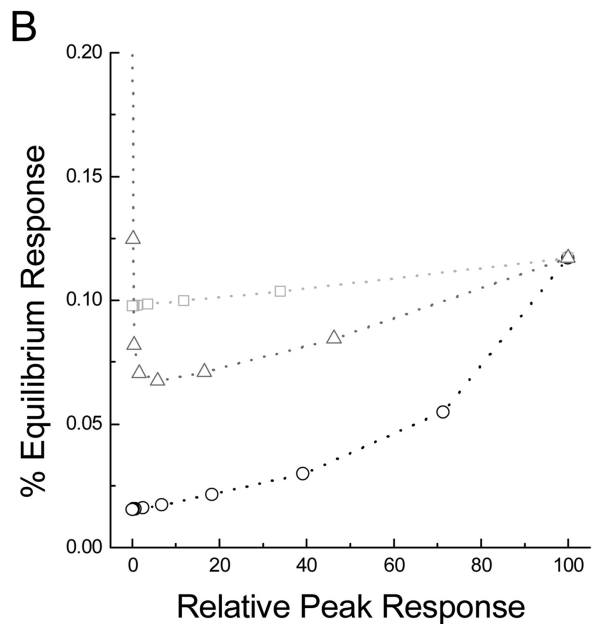

Multi-open state model

Pre-gating model

Figure 8. The equilibrium/peak relationship is reproduced by reducing the stability of the closed-bound state. $A$, Structures of the kinetic schemes from Bowie et al. (1998) (left), Perrais et al. (2009) (middle), and Zhang et al. (2008) (right) analyzed in $\boldsymbol{B}$. B , Results of changing a single parameter (either $\alpha, \beta, \delta$, or $(0)$ on the relationship between peak and equilibrium activation for the above models. Only alterations to CO reproduced the relationship.

this relationship, whereas models lacking them fail to do so. Second, the chemical nature of the cation bound to the Met770 site further regulates the responsiveness to KAR agonists. This effect is not observed with anion substitutions despite close physical and functional association between anion and cation-binding sites. This finding, therefore, is the first experimental dissociation between anion and cation effects on KARs. It also underscores an emerging idea that cations exert a more prominent role on the gating process than anions (Bowie, 2010). We therefore propose that a key determinant of KAR agonist efficacy is the relative stability of pregating conformations, a mechanism regulated by both the agonist molecule and external cations.

Why do cations but not anions alter relative agonist efficacy? The original study identifying cation and anion effects yielded the surprising result that ions of opposite charge modulate KARs in an apparently identical manner (Bowie, 2002). This finding was puzzling as it seemed to favor a common site of action for ions of 
different valency while defying the normal dictate of electrostatic principles (Bowie, 2010). The conundrum was eventually resolved, however, with structural and functional data showing that anion- and cation-binding sites are physically discrete but functionally coupled (Plested and Mayer, 2007; Wong et al., 2007; Plested et al., 2008; Bowie, 2010). Building on this idea, it was subsequently proposed that anions and cations affect KARs in an identical fashion by regulating the stability of the subunit dimer interface (Chaudhry et al., 2009), a structure thought to regulate desensitization (but see Bowie, 2010). Anions may do this directly by bridging the dimer interface. Cations may also do this directly through water-mediated contact with the opposing dimer (Plested and Mayer, 2007; Plested et al., 2008; Bowie, 2010) or indirectly by influencing anion residency time (Vijayan et al., 2009). Our data suggest that in addition to influencing the dimer, cations also affect GluK2 ABD closed-cleft stability accounting for their singular effect on GluK2 receptors.

\section{On the nature of partial agonism at kainate receptors}

Structure-function studies of iGluR agonist behavior were only made possible after the isolated $\mathrm{ABD}$ structure of each receptor subclass was solved (Armstrong et al., 1998; Armstrong and Gouaux, 2000; Inanobe et al., 2005; Mayer, 2005; Nanao et al., 2005; Naur et al., 2007). With structures at hand, AMPAR agonist efficacy was proposed to be dictated by conformations adopted by the ABD. Full agonists were considered more effective because they promoted greater domain closure than partial agonists (Armstrong and Gouaux, 2000; Jin et al., 2003). A similar mechanism was then proposed for KARs (Mayer, 2005; Nanao et al., 2005; Hald et al., 2007). An important caveat, however, was that only a few ligand-bound KAR structures were ever analyzed. When a more extensive range of full and partial agonists was eventually examined, a clear relationship between efficacy and the ABD closure was not observed (Fay et al., 2009; Frydenvang et al., 2009). This finding alone suggested the need to identify models of agonist efficacy that would explain published data. An added complication is that KAR activation is regulated by external anions and cations (Bowie, 2002, 2010). Consequently, any new model would need to consider the individual and added contributions of the agonist and external ions.

Here we have developed a new working model of agonist behavior at GluK2 KARs. From analysis of a range of ligands, we show that the degree of macroscopic desensitization is proportional to agonist efficacy. GluK2 receptor responses decline almost completely in the presence of full agonists but only moderately with partial agonists. This finding is unexpected for two reasons. First, coupling between agonist efficacy and macroscopic desensitization is not at all predicted from our current understanding of KAR gating. In support of this, simulations with two commonly used KAR gating schemes failed to establish any correlation between these properties. Second, this relationship makes the surprising prediction that, at its most extreme, the "perfect" partial agonist would elicit a response that lacks macroscopic desensitization. Our dataset does not provide experimental support for this idea since all partial agonist responses examined decayed to some extent. However, a nondesensitizing phenotype has been reported when mutations are introduced into the dimer interface of non-NMDARs (Armstrong et al., 2006; Weston et al., 2006; Nayeem et al., 2009).

Could mutations that render receptors nondesensitizing (or nondecaying) also convert full agonists to partial agonists? It turns out this might be the case. In their study of AMPAR and KAR dimer mutants, Weston et al. (2006) noted that the response amplitude of the "nondesensitizing" AMPAR was enhanced by cyclothiazide (see Fig. $6 d$ of study), a thiazide diuretic that blocks microscopic and macroscopic desensitization (Rosenmund et al., 1998). Although, the authors even make the point that L-Glu now acts as a partial agonist, they still concluded that these dimer mutations genuinely blocked desensitization (Weston et al., 2006). A point not considered in study is that both receptor families gate ion channels with multiple open states (Howe, 1996; Swanson et al., 1996, 1997). For example, a possible explanation is that the conductance state(s) that gives rise to the peak response is distinct from that accounting for the equilibrium response (Bowie and Lange, 2002). In fact, the possibility that peak and equilibrium responses represent distinct states of the GluK2 ion channel is supported in three ways. First, the plant lectin, concanavalin-A, modulates only equilibrium and not peak GluK2 responses (Bowie et al., 2003). Second, nondesensitizing mutant GluK2 receptors are insensitive (or weakly sensitive) to external anions and cations (Plested et al., 2008), whereas peak responses are wholly dependent on them (Wong et al., 2006). Finally, the main open state of GluK2 peak responses is about 27 pS (Zhang et al., 2009), whereas steady-state KAR unitary events are apparently much smaller at about 5 pS (Howe, 1996; Swanson et al., 1996). Together, this added complexity provides a much more realistic framework where agonist efficacy is determined by how often full and partial agonists access distinct conductance states.

\section{Pregating transitions as a common mechanism of partial agonism}

The separation of binding and gating events by del Castillo and Katz (1957) has remained the framework for partial agonism for $>50$ years. They proposed that while full agonists efficiently proceed from the bound, nonconducting state to the open state, partial agonists do so less frequently, resulting in lower efficacy. Recent studies of Cys-loop receptors have argued that, in fact, this final transition to the open state is independent of the agonist type (Lape et al., 2008; Mukhtasimova et al., 2009). Rather, the crucial determinant in agonist efficacy is an additional step (or steps) imposed between the initial binding step and final gating isomerization that forms the pregating conformation, referred to as either a flipped (Lape et al., 2008) or a primed (Mukhtasimova et al., 2009) state. Full agonists promote the formation of this pregating state allowing the final step of opening to proceed, whereas partial agonists are not as effective at stabilizing these conformations.

Are effects on pregating conformations also important for agonist behavior at iGluRs? Similar studies on NMDARs that fit single-channel records to a reaction scheme have reached opposing conclusions, with one group finding that partial agonists modify only pregating transitions (Erreger et al., 2005) while another study found that different agonists affect different rates within the gating scheme (Kussius and Popescu, 2009; Kussius et al., 2010). Fitting single-channel records to gating schemes has yet to be applied to KARs. Consequently, it is unclear whether differences in agonist efficacy occurs at the level of the open/shut reaction, as originally proposed by del Castillo and Katz (1957), at the pregating level, as in Cys-loop receptors (Lape et al., 2008; Mukhtasimova et al., 2009), or at all levels as is the case for some NMDARs (Kussius and Popescu, 2009). Despite this uncertainty, there is evidence suggesting that the relative stability or occupancy of pregating states plays a role in determining agonist efficacy, at least for AMPARs. Here it has been argued that the pregating state corresponds to the ABD closed-cleft conforma- 
tion and point mutations that destabilize the closed conformation also reduce agonist efficacy (Robert et al., 2005; Zhang et al., 2008). Our simulations with gating models (Fig. 8) suggest that the pregating transition is the crucial factor underlying the inverse relationship between peak and equilibrium responses for an extended series of KAR agonists. Given the importance of the pregating closed-cleft conformation, it would be interesting in future work to determine whether this relationship is also regulated by the type of monovalent cation present in the external milieu.

\section{References}

Armstrong N, Gouaux E (2000) Mechanisms for activation and antagonism of an AMPA-sensitive glutamate receptor: crystal structures of the GluR2 ligand binding core. Neuron 28:165-181.

Armstrong N, Sun Y, Chen GQ, Gouaux E (1998) Structure of a glutamatereceptor ligand-binding core in complex with kainate. Nature 395: 913-917.

Armstrong N, Jasti J, Beich-Frandsen M, Gouaux E (2006) Measurement of conformational changes accompanying desensitization in an ionotropic glutamate receptor. Cell 127:85-97.

Barberis A, Sachidhanandam S, Mulle C (2008) GluR6/KA2 kainate receptors mediate slow-deactivating currents. J Neurosci 28:6402-6406.

Birdsey-Benson A, Gill A, Henderson LP, Madden DR (2010) Enhanced efficacy without further cleft closure: reevaluating twist as a source of agonist efficacy in AMPA receptors. J Neurosci 30:1463-1470.

Bowie D (2002) External anions and cations distinguish between AMPA and kainate receptor gating mechanisms. J Physiol 539:725-733.

Bowie D (2010) Ion-dependent gating of kainate receptors. J Physiol 588:67-81.

Bowie D, Lange GD (2002) Functional stoichiometry of glutamate receptor desensitization. J Neurosci 22:3392-3403.

Bowie D, Lange GD, Mayer ML (1998) Activity-dependent modulation of glutamate receptors by polyamines. J Neurosci 18:8175-8185.

Bowie D, Garcia EP, Marshall J, Traynelis SF, Lange GD (2003) Allosteric regulation and spatial distribution of kainate receptors bound to ancillary proteins. J Physiol 547:373-385.

Chaudhry C, Plested AJ, Schuck P, Mayer ML (2009) Energetics of glutamate receptor ligand binding domain dimer assembly are modulated by allosteric ions. Proc Natl Acad Sci U S A 106:12329-12334.

Del Castillo J, Katz B (1957) Interaction at end-plate receptors between different choline derivatives. Proc R Soc Lond B Biol Sci 146:369-381.

Erreger K, Geballe MT, Dravid SM, Snyder JP, Wyllie DJ, Traynelis SF (2005) Mechanism of partial agonism at NMDA receptors for a conformationally restricted glutamate analog. J Neurosci 25:7858-7866.

Fay AM, Bowie D (2006) Concanavalin-A reports agonist-induced conformational changes in the intact GluR6 kainate receptor. J Physiol 572:201-213.

Fay AM, Corbeil CR, Brown P, Moitessier N, Bowie D (2009) Functional characterization and in silico docking of full and partial GluK2 kainate receptor agonists. Mol Pharmacol 75:1096-1107.

Fleck MW, Cornell E, Mah SJ (2003) Amino-acid residues involved in glutamate receptor 6 kainate receptor gating and desensitization. J Neurosci 23:1219-1227.

Frydenvang K, Lash LL, Naur P, Postila PA, Pickering DS, Smith CM, Gajhede M, Sasaki M, Sakai R, Pentikainen OT, Swanson GT, Kastrup JS (2009) Full domain closure of the ligand-binding core of the ionotropic glutamate receptor iGluR5 induced by the high affinity agonist dysiherbaine and the functional antagonist 8,9-dideoxyneodysiherbaine. J Biol Chem 284:14219-14229.

Hald H, Naur P, Pickering DS, Sprogoe D, Madsen U, Timmermann DB, Ahring PK, Liljefors T, Schousboe A, Egebjerg J, Gajhede M, Kastrup JS (2007) Partial agonism and antagonism of the ionotropic glutamate receptor iGLuR5: structures of the ligand-binding core in complex with domoic acid and 2-amino-3-[5-tert-butyl-3-(phosphonomethoxy)-4isoxazolyl]propionic acid. J Biol Chem 282:25726-25736.

Howe JR (1996) Homomeric and heteromeric ion channels formed from the kainate-type subunits GluR6 and KA2 have very small, but different, unitary conductances. J Neurophysiol 76:510-519.

Inanobe A, Furukawa H, Gouaux E (2005) Mechanism of partial agonist action at the NR1 subunit of NMDA receptors. Neuron 47:71-84.

Jin R, Banke TG, Mayer ML, Traynelis SF, Gouaux E (2003) Structural basis for partial agonist action at ionotropic glutamate receptors. Nat Neurosci 6:803-810.

Kussius CL, Popescu GK (2009) Kinetic basis of partial agonism at NMDA receptors. Nat Neurosci 12:1114-1120.

Kussius CL, Popescu AM, Popescu GK (2010) Agonist-specific gating of NMDA receptors. Channels (Austin) 4:78-82.

Lape R, Colquhoun D, Sivilotti LG (2008) On the nature of partial agonism in the nicotinic receptor superfamily. Nature 454:722-727.

Mayer ML (2005) Crystal structures of the GluR5 and GluR6 ligand binding cores: molecular mechanisms underlying kainate receptor selectivity. Neuron 45:539-552.

Mukhtasimova N, Lee WY, Wang HL, Sine SM (2009) Detection and trapping of intermediate states priming nicotinic receptor channel opening. Nature 459:451-454.

Nanao MH, Green T, Stern-Bach Y, Heinemann SF, Choe S (2005) Structure of the kainate receptor subunit GluR6 agonist-binding domain complexed with domoic acid. Proc Natl Acad Sci U S A 102:1708-1713.

Naur P, Hansen KB, Kristensen AS, Dravid SM, Pickering DS, Olsen L, Vestergaard B, Egebjerg J, Gajhede M, Traynelis SF, Kastrup JS (2007) Ionotropic glutamate-like receptor delta2 binds D-serine and glycine. Proc Natl Acad Sci U S A 104:14116-14121.

Nayeem N, Zhang Y, Schweppe DK, Madden DR, Green T (2009) A nondesensitizing kainate receptor point mutant. Mol Pharmacol 76:534-542.

Paternain AV, Cohen A, Stern-Bach Y, Lerma J (2003) A role for extracellular $\mathrm{Na}+$ in the channel gating of native and recombinant kainate receptors. J Neurosci 23:8641-8648.

Perrais D, Coussen F, Mulle C (2009) Atypical functional properties of GluK3-containing kainate receptors. J Neurosci 29:15499-15510.

Plested AJ, Mayer ML (2007) Structure and mechanism of kainate receptor modulation by anions. Neuron 53:829-841.

Plested AJ, Vijayan R, Biggin PC, Mayer ML (2008) Molecular basis of kainate receptor modulation by sodium. Neuron 58:720-735.

Robert A, Armstrong N, Gouaux JE, Howe JR (2005) AMPA receptor binding cleft mutations that alter affinity, efficacy, and recovery from desensitization. J Neurosci 25:3752-3762.

Rosenmund C, Stern-Bach Y, Stevens CF (1998) The tetrameric structure of a glutamate receptor channel. Science 280:1596-1599.

Siso-Nadal F, Ollivier JF, Swain PS (2007) Facile: a command-line network compiler for systems biology. BMC Syst Biol 1:36.

Swanson GT, Feldmeyer D, Kaneda M, Cull-Candy SG (1996) Effect of RNA editing and subunit co-assembly single-channel properties of recombinant kainate receptors. J Physiol 492:129-142.

Swanson GT, Kamboj SK, Cull-Candy SG (1997) Single-channel properties of recombinant AMPA receptors depend on RNA editing, splice variation, and subunit composition. J Neurosci 17:58-69.

Vijayan R, Plested AJ, Mayer ML, Biggin PC (2009) Selectivity and cooperativity of modulatory ions in a neurotransmitter receptor. Biophys J 96:1751-1760.

Weston MC, Schuck P, Ghosal A, Rosenmund C, Mayer ML (2006) Conformational restriction blocks glutamate receptor desensitization. Nat Struct Mol Biol 13:1120-1127.

Wong AY, Fay AM, Bowie D (2006) External ions are coactivators of kainate receptors. J Neurosci 26:5750-5755.

Wong AY, MacLean DM, Bowie D (2007) $\mathrm{Na}+/ \mathrm{Cl}$ - dipole couples agonist binding to kainate receptor activation. J Neurosci 27:6800-6809.

Zhang W, Robert A, Vogensen SB, Howe JR (2006) The relationship between agonist potency and AMPA receptor kinetics. Biophys J 91:1336-1346.

Zhang W, Cho Y, Lolis E, Howe JR (2008) Structural and single-channel results indicate that the rates of ligand binding domain closing and opening directly impact AMPA receptor gating. J Neurosci 28:932-943.

Zhang W, St Gelais F, Grabner CP, Trinidad JC, Sumioka A, MorimotoTomita M, Kim KS, Straub C, Burlingame AL, Howe JR, Tomita S (2009) A transmembrane accessory subunit that modulates kainate-type glutamate receptors. Neuron 61:385-396. 